\title{
Multisteps Sales Forecasting using LSTM Compared to GMDH
}

\author{
Ghita Rguiga \\ LRI Laboratory - ENSEM Casablanca \\ Hassan II University
}

\author{
Jamal Benhra \\ LRI Laboratory - NSEM Casablanca \\ Hassan II University
}

\begin{abstract}
This work reports the effectiveness of using Recurrent Neural Network LSTMs for multistep sales forecasting instead of a standalone approach and the use of time series data, treatment of inflation, forecasting options and tunings to help decision executives make better decisions on forecast and management systems. This work is lead on historical sales based on a year of demand records, the program established using LSTMs showed minimal loss and optimal forecasts compared to prediction using GMDH which is known for management forecasts such as inventories and sales forecasting.
\end{abstract}

\section{General Terms}

Supply Chain Management, Sales Forecasting, Neural Networks for Prediction, LSTM, RNN, GMDH Projects, Multi Echelon Prediction

\section{Keywords}

LSTM, RNN, GMDH, Sales Forecasting, Artificial Intelligence, Supply Chain Management

\section{INTRODUCTION}

Forecasting helps to anticipate potential problems. For example, if a team only reaches $35 \%$ of its quota, it is possible to analyze the situation to consider solutions. Perhaps a competitor has aggressively lowered prices or the company's new business strategy been producing unexpected side effects. The earlier a problem is discovered, the easier it is to solve it.

Sales forecasting influences many decisions, from recruiting to resource management to goal setting and budgeting. For example, if the forecast predicts $26 \%$ more sales opportunities, recruitment must be considered to meet demand. If, on the other hand, a decline in activity is anticipated, human resources must be managed accordingly. In addition, it may be wise to invest time in marketing and prospecting to counter this decline. [1]

Digital technologies such as advanced Point-of-Sale (POS) systems, the "Internet of Things" (IoT), and cloud computing have enabled firms to collect vast amounts of data in real time. Then these vast amounts of data that are collected and analyzed in near real-time have the potential to improve our understanding of customer behavior, improve demand prediction and better execute supply chain management [2]

Since sales forecasting has become a prerequisite for successful management. This work reports the effectiveness of using Recurrent Neural Network LSTMs for multistep sales forecasting instead of using a standalone approach, use of time series data, treatment of inflation, forecasting options and programs, and management of forecasting systems.

This paper is presented in several sections. The following section presents a review of previous works on the use of data analysis techniques for sales forecasting. The case study and the analyzed data are described in the third section. The Methodology section provides a description of the methodology followed and the performance evaluation criteria applied. The fifth section presents all the results obtained as well as a discussion focused on these results, and finally, the conclusions of the work are presented.

\section{LITERATURE REVIEW}

To explore the issue of sales forecasting, several projects have been developed by researchers over the years, however, to the best of knowledge, the application of deep learning algorithms in sales forecasting using a multistep approach is still incipient with a very limited number of published works that we are going to cite in this paper.

A study of Machine-Learning Models for Sales Time Series Forecasting was conducted with a main goal considering approaches and case studies of using machine learning for sales forecasting. The effect of machine-learning generalization has also been considered. This effect can be used to make sales predictions when there is a small amount of historical data for specific sales time series in the case when a new product or store is launched. A stacking approach for building regression ensemble of single models has been studied. The results show that the performance of predictive models for sales time series forecasting can be improved by using stacking techniques. [3]

Furthermore, a research on Sales Prediction through GMDH for a Small Dataset has shown the applicability of GMDH Methods in a case where a company reports a short timeseries given the changes in its warehouse structure. Given the right inventory using the company revenues, it uses a. thus, learning rates variations do not significantly increase the computing time, and the validation fails with an error minor to five percent which is a high standard.[4]

Then a work was conducted on Sales Forecasting using RNN which explains the concept of the system architecture of sales forecasting using recurrent neural networks with variant features, the foremost part is based on sales propelled by the sales administrator of a company. Managing the information on the stock and updating the stock value. The generality of RNN is the continuous form of the working part. It is discreetly used for the sales updating norms, amount of product that has been produced, and the wide continuity of product sales in the previous year's till on to the date. the accuracy of these sales forecasting enables companies to take important business decisions and can improve in their performance for the profitable sales, the LSTM (Long Short Term Memory) in this work has been used for classifying, processing and making predictions along with the time series, by which the products are been checked for the sales forecasting and reported by checking up with the time series of previous year's sales of the already existing products, this 
is the continuous range of checking up the product that is sold that this work has proposed. [5]

In our knowledge, these are the works already lead on prediction of sales, therefore these works are still limited since the first study is issued by using a small dataset which makes the data non representative, also as mentioned on the second study, checks are based on the same period of the previous year which doesn't tolerate evolution and change sets, the third study performs on new products which means that there is no history data, also the use of simple regression limits its performance.

\section{STUDY CONTEXTUALIZATION}

Good sales forecasting helps businesses to grow, but, for many years, forecasting has relied on the human element, emotions and hunches that can make or break a quarter. Just as big data and artificial intelligence (AI) pervade many aspects of how we work, it is doing the same for forecasting. AI equips businesses with improved lead scoring capabilities, enabling them to determine the fate of sales opportunities more accurately, and, ultimately, produce more accurate sales forecasts. AI can sift through large volumes of real-time and historical data and identify the most lucrative sales leads. Using intricate algorithms, AI can fine-tune lead scores by accounting for a multitude of different factors. [6]

Also, AI is driven by machine learning, which means the software trains on data and becomes better over time.

However classical models have a significant dependence on statistical assumptions, which diminish their capacity to predict a behavior that was not previously registered. To deal with such problem, Artificial Neural Networks (ANNs) appear as a suitable alternative. These techniques try to mimic neural cells behavior, which makes them non-linear models by nature. Because ANNs are data-driven, they have a selfadaptive feature. This adaptation capacity makes them suitable to analyze problems where data is not complete, or when its documentation was not appropriately gathered (i.e., data with noise). Given that ANNs allow the approximation of almost all non-linear continuous function, they are suitable for sales forecasting. However, in the classical literature, there is not a unique approach to sales forecasting because ANNs may have different structures according to the problem. For example, electricity demand forecasting considers a hybrid structure since data may present seasonal effects, Yet, it will take both the human and technological sides working together to achieve true success. [7]

A Recurrent Neural Network is basically a standard neural network that has been extended across time by having edges which feed into the next time step instead of into the next layer in the same time step. RNN is designed to recognize sequences, for example, a speech signal or a text. It has cycles inside that implies the presence of short memory in the net

One of the issues of the recurrent neural network is related to sequence as a direct cycle is formed with their connections. The next state can be retained using the current output as input for the next step resembling as running a program with standard inputs and selected internal variables (figure 3 ).

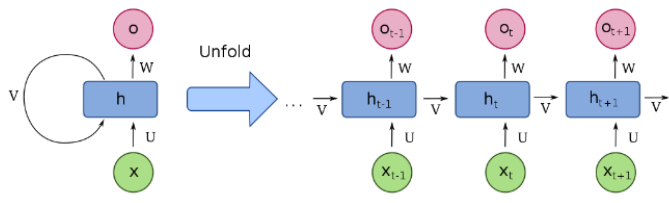

Figure 1. Recurrent Neural Network graphic

Long Short-Term Memory usually just called "LSTMs" (figure 2) - are a special kind of RNN, capable of learning long-term dependencies. They were introduced by Hoch Reiter \& Schmid Huber (1997) and were refined and popularized by many people in following work. They are widely used on a large set of issued and designed specifically to eliminate long-term dependency. The default behavior LSTM can capture and store information for a longer span of time. The main component of LSTM is the cell state, a horizontal line that keeps running all through. The cell state always runs down the entire chain with less frequent interactions. It is very easy for information to just flow unchanged along with it. LSTM has all the rights to include or exclude the information to the cell state that are let through by structures called gates. It is a way to decide whether to let the information through or not. It consists of a sigmoid network layer and performs multiplication operation. The output value of the sigmoid layer ranges between zero and one indicating the individual component to decide whether to go through or not. A value zero indicates "let nothing through" and one indicates "let everything through". [8][9]

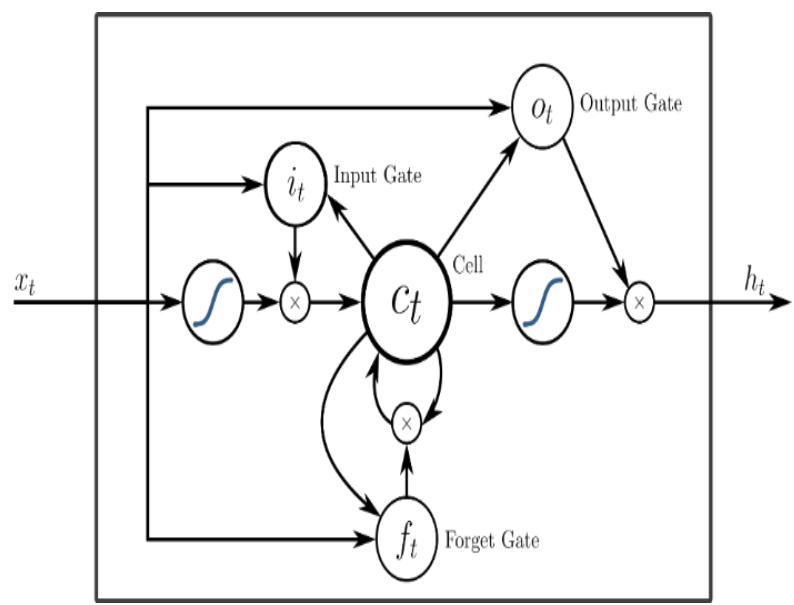

Figure 2. The structure of an LSTM unit

\section{PROBLEM DEFINITION}

In this work we are going to present a method for double step sales forecasting using Recurrent Neural Networks LSTMs based on a data set recorded from a CRM, in this section we are going to present some analysis of the data chosen for experimentation, then the results given by each method of prediction, a comparative study is leaded as well.

\subsection{Data preparation}

Our dataset represents one-year record of a B2C organization's sales for multiple products, the references have been anonymized to protect personal data, privacy, and the legitimate interest of legal persons. 


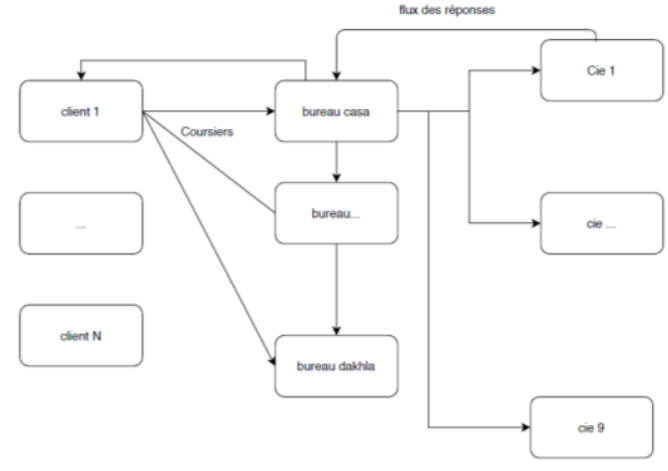

Figure 1. Synoptic Schema of data flow

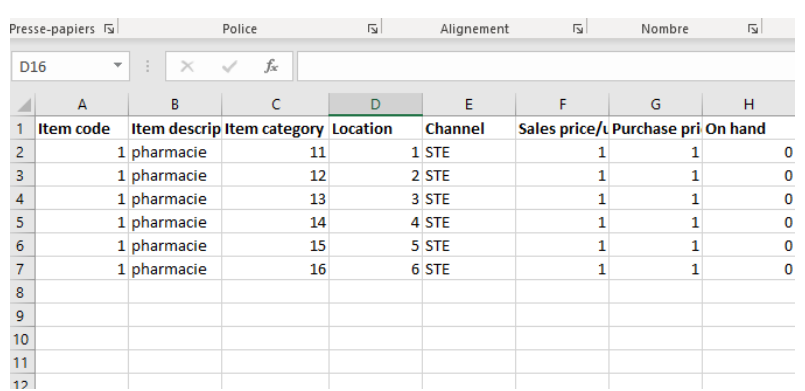

Figure 4. Data recorded

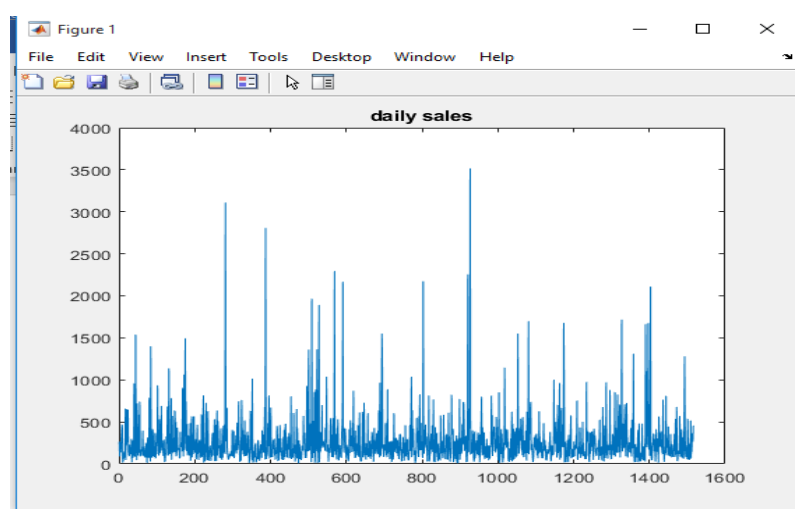

Figure 2. Data Visualization using MATLAB plot tool

The data set has been standardized to get a time series based on the total amount of daily sales, the analytics have shown a huge variation and no trending which makes sense for experimenting prediction.

\subsection{Training methods for Deep Learning Networks used:}

There is an abundant amount of great knowledge about deep learning, here are specified the powerful deep learning methods that are applied to our forecasting problem.

\section{Back-Propagatio [11]}

Back-prop is simply a method to compute the partial derivatives (or gradient) of a function, which has the form as a function composition (as in Neural Nets). When you solve an optimization problem using a gradient-based method (gradient descent is just one of them), you want to compute the function gradient at each iteration.

\section{Levenberg-Marquardt Algorithm}

The Levenberg-Marquardt algorithm, also known as the damped least-squares method, has been designed to work specifically with loss functions which take the form of a sum of squared errors. It works without computing the exact Hessian matrix. Instead, it works with the gradient vector and the Jacobian matrix. Consider a loss function which can be expressed as a sum of squared errors of the form.

Long Short-Term Memory:

The LSTM network has the following three aspects that differentiate it from a usual neuron in a recurrent neural network:

- It has control on deciding when to let the input enter the neuron.

- It has control on deciding when to remember what was computed in the previous time step.

- It has control on deciding when to let the output pass on to the next time stamp.

- The beauty of the LSTM is that it decides all this based on the current input itself. [12]

\subsection{Data processing}

At first we experimented prediction with an LSTM network applied to the first step of data then as the prediction is recorded, the second step is also predicted by an LSTM network, and since we are based on a time series dataset, a closed loop network has also been experimented considering sequences relationships, last method we used is a recurrent neural network with two LSTM layers, then we proceeded to prediction using projects method on GMDH to predict sales and inventory, we plotted both results and compared KPIs. [10]

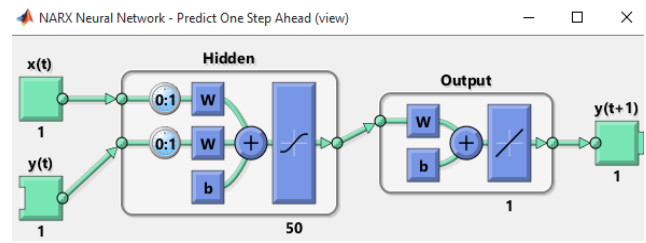

Figure 6. Data injected on GMDH

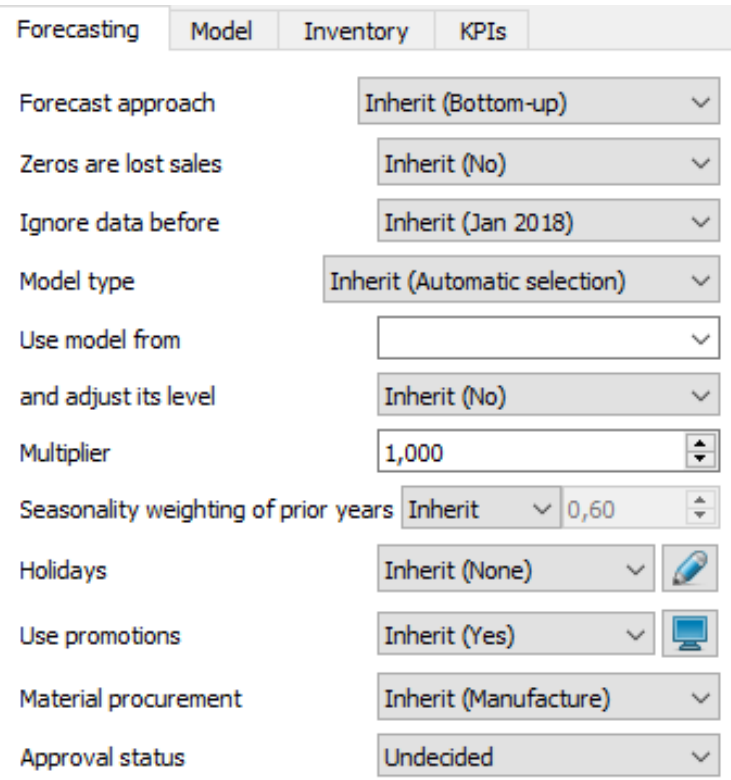

Figure 7. GMDH Tunning 


\subsection{Results}

After training the programs we plotted the original targets, the network predictions and the expected data to show the performance on the results, therefore, the training of the simple network (figure) and the recurrent(figure) one has performed well but not reliable on some periods as shown on the figures.
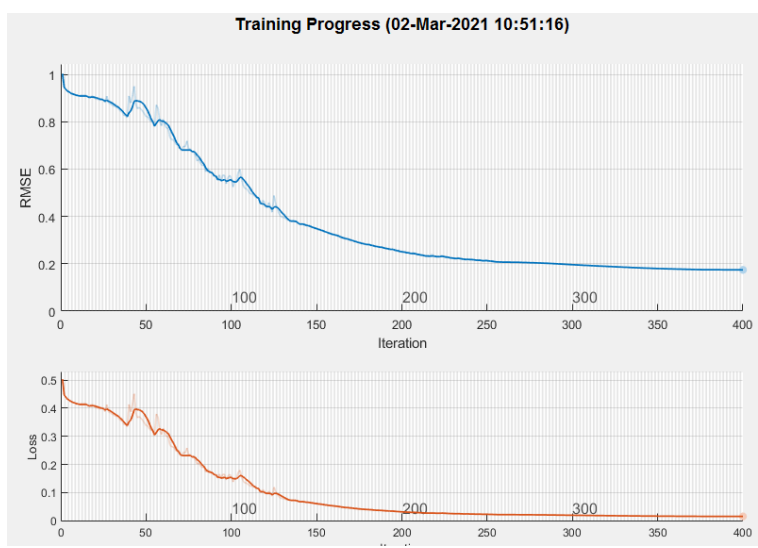

Figure 8. Prediction progress using LSTM
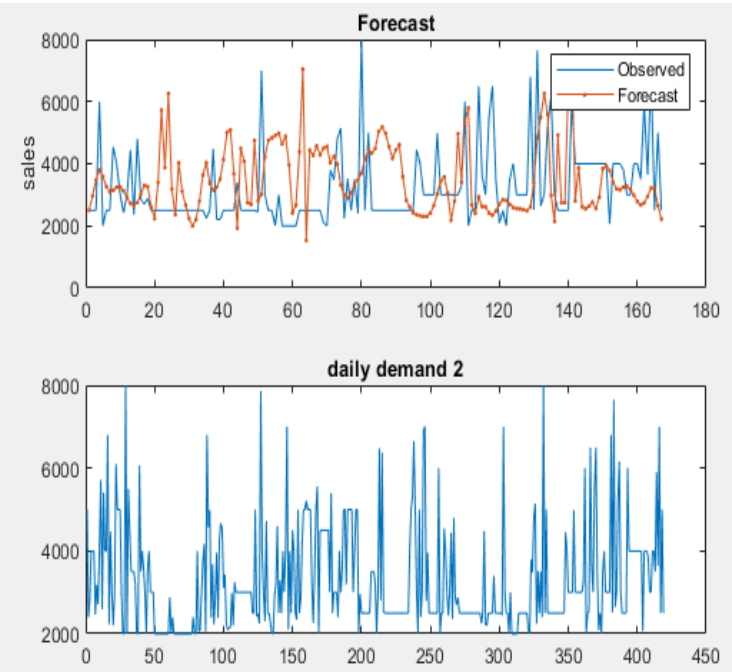

Figure 9 Data Comparison

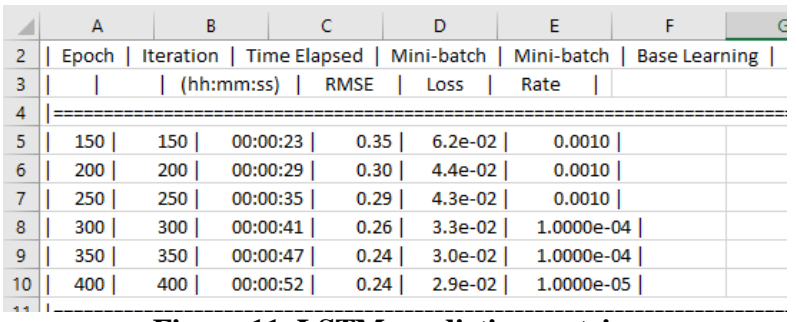

Figure 11. LSTM prediction metrics

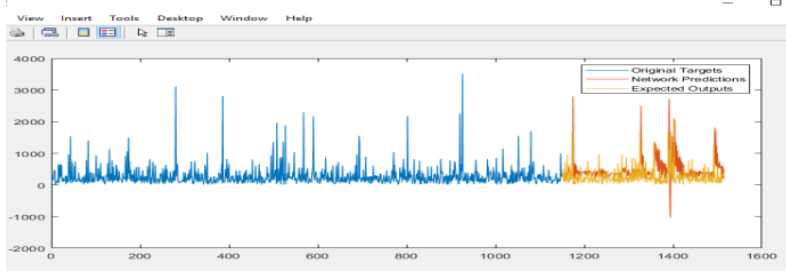

Figure 12. LSTM Prediction
As shown on figures 8 to 12 , LSTM prediction has performed well and gave results near the observed data with a loss of 0.1 and an RMSE of 0.3 , this has been applied to 6 months data to predict the first step demand and to 4 months to predict the second step, the program performs on both steps with a reliable efficiency.

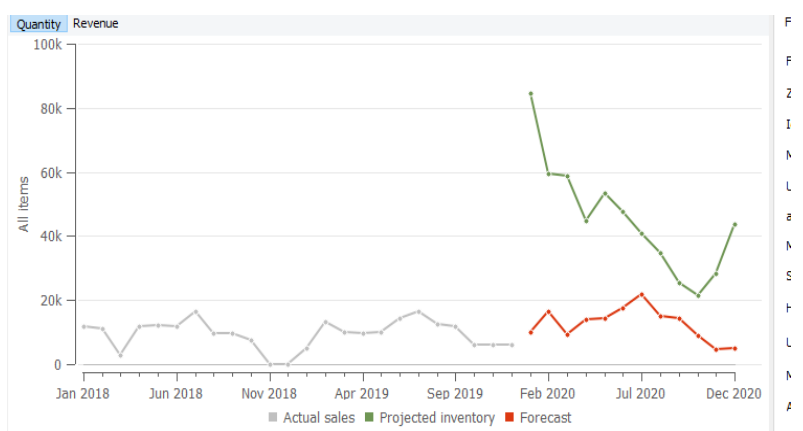

Figure 13. GMDH prediction

\begin{tabular}{|c|c|c|c|}
\hline \multirow[t]{2}{*}{ Forecasting } & \multirow[t]{2}{*}{ Model } & inventory & \multirow{2}{*}{$\begin{array}{l}\text { KPIs } \\
\text { Value }\end{array}$} \\
\hline & & & \\
\hline \multicolumn{2}{|c|}{ Inventory value } & \multicolumn{2}{|c|}{788440,97} \\
\hline \multicolumn{4}{|c|}{ Days of supply } \\
\hline \multicolumn{2}{|c|}{ Expected overstock value } & \multicolumn{2}{|c|}{425440,63} \\
\hline \multicolumn{2}{|c|}{ Expected stockout value } & \multicolumn{2}{|c|}{70061,25} \\
\hline \multicolumn{2}{|c|}{ Non-moving inventory value } & \multicolumn{2}{|l|}{0} \\
\hline \multicolumn{2}{|c|}{ Turnover| Turns/year } & \multicolumn{2}{|l|}{0,04} \\
\hline \multicolumn{2}{|c|}{ Turnover | Days to sell } & \multicolumn{2}{|c|}{9727} \\
\hline \multicolumn{2}{|c|}{ Gross margin } & \multicolumn{2}{|c|}{$12,5 \%$} \\
\hline \multicolumn{2}{|c|}{ Turn-earn index } & \multicolumn{2}{|l|}{0,5} \\
\hline \multicolumn{2}{|c|}{ Annual revenue } & \multicolumn{2}{|c|}{1336999,41} \\
\hline \multicolumn{2}{|c|}{ Next year revenue } & \multicolumn{2}{|c|}{1002287,98} \\
\hline \multicolumn{2}{|l|}{ Annual sales } & \multicolumn{2}{|c|}{121656} \\
\hline \multicolumn{2}{|c|}{ Next year sales } & \multicolumn{2}{|c|}{91200} \\
\hline
\end{tabular}

Figure 14. GMDH KPIs

As shown on figures 13 to 16 GMDH has performed as a step by step, thus, GMDH is yet a production planner that works for inventories and planning based on records, it is not adapted to multistep predictions.

\section{RESULTS DISCUSSION}

As shown on the previous sections, this work consists of predicting sales based on demand and productivity, the figure 12 shows that loss is nearby 0.1 for the LSTM multistep forecast, therefore, also GMDH has performed well on both datasets, yet GMDH is better used for inventory forecasts based on revenue and quotas, LSTM has shown to be performing better in this case and offers a parallel prediction on both sets, which allows to lead preventive planning actions for brokers.

Our perspective of this work is to provide a reliable prediction system that offers real-time management decisions and help decision makers plan resources according to demand prediction.

\section{REFERENCES}

[1] Nilanjan Dey, Aboul Ella Hassanien,2017, Internet Of Things And Big Data Analytics Toward Next-Generation Intelligence 
[2] Big Data Challenges and Issues: Review on Analytic Technique - Issn: 0976-5166 - Tannu Et Al. / Indian Journal of Computer Science and Engineering (Ijcse) Jul 2017

[3] Ghita.R, Jamal.B, 2019, Sales Forecasting Using Lstm In the Context of a Moroccan Company - Mosim20, Conference Paper

[4] Dimitr.is,2019, Energy Demand Prediction Using GMDH Networks

[5] Kalaiarasan T R, Anandkumar V, The International Journal of Innovative Technology and Exploring Engineering (Ijitee) Issn: 2278-3075, Volume-8 Issue-9, July 2019, Sales Forecasting Using RNN

[6] Ai,Salim.B - December 2017 - international Journal of Interactive Multimedia And Artificial Intelligence - Doi: 0.9781/ Jjimai.2018.04.003, Innovate Your Strategy
[7] Ghita.R, Mosim20,2020, Sales Forecasting Based on LSTM In the Context of A Moroccan Company

[8] Alibpage , on August 27, 2015, Understanding LSTM Networks.

[9] S. J. Farlow, 2017, Self-Organizing Methods in Modeling: GMDH Type Algorithms - ISBN 9780824771614 - Published July 11, 1984 by CRC Press

[10] Dipti Srinivasan, Neurocomputing journal, 2008, Energy demand prediction using GMDH networks

[11] Pen Treebank, Pen.T,2018, Language Modelling

[12] Jaber.F, Ghita.R, Journal of operations management, optimization and decision support, ISSN: 2658-9141 JOMODS 2020, Forecasting Demand based on RNN. 